\title{
Efecto del Calcio y Azufre en el Sistema Maíz - Frijol en Ladera ante un Nivel de Nitrógeno y Fósforo en El Salvador 19891
}

\author{
José Heriberto Sosa M., Víctor M. Mendoza O, Edgar Noel Ascencio, Ana Gertrudis Alvarado2, \\ Sonia Bonilla ${ }^{3}$ y William R. Raun 4
}

\section{COMPENDIO}

Dos ensayos fueron iniciados por dos años en suelos derivados de ceniza volcanica en Opico Quezaltepeque, El Salvador, para evaluar la respuesta de aplicar azufre $(\mathrm{S})$, calcio $(\mathrm{Ca})$ y $(\mathrm{K})$ potasio en un sistema maíz-frijol (Maíz sembrado en mayo, frijol sembrado en relevo en agosto). También se evaluó el efecto de quemar los residuos lo cual es una práctica común, en comparación a la labranza cero. Cuatro ciclos de datos ( 2 de maíz y 2 de frijol) fueron obtenidos en las dos localidades. Los tratamientos consistieron en varias combinaciones de sulfato de amonio y urea, con y $\sin \mathrm{S}$, Ca y K.

Los rendimientos fueron mayores cuando se aplicó sulfato de amonio (100 kg N/ha) junto con formula (16-20-0,30 $\mathrm{kg} \mathrm{P}_{2} \mathrm{O}_{5} /$ ha) en bandas en labranza cero. Este mismo tratamiento evaluado con la quema daba rendimientos significativamente mas bajos. Comparando sulfato de amonio y urea aplicados juntos con el fósforo se notó una posible respuesta de azufre en estos suelos. Aplicaciones de potasio no produjeron incremento en el rendimiento de maíz o frijol. La respuesta de haber aplicado $3 \mathrm{Mg}$ de cal dolomítica / ha en los dos ciclos de maíz resultó en incrementos en el rendimiento del frijol; pero esto se observó hasta el último ciclo.

Palabras claves adicionales: labranza, fertilidad.

\section{INTRODUCCIÓN}

En el área de Opico Quezaltepeque, El Salvador, se siembra el maíz (Zea mays L.) predominantemente en terrenos de ladera donde es común la práctica de la quema de rastrojos del año anterior por lo cual los suelos quedan desprotegidos y susceptibles a la erosión. Estudios previos en esta área han definido la erosión y la fertilidad de los suelos como factores limitantes (Mendoza y Sosa, 1983). Varios trabajos han señalado deficiencias de azufre (S) en suelos volcánicos (Fitts, 1970). Aunque, la eficiencia del uso de S haya sido afectada por interacciones antagónicas con el fósforo (P) y el Ca (Barrow, 1969, Ensminger, 1954, Kamprath et al., 1956). También se ha notado que las deficiencias de $\mathrm{S}$ se pueden encontrar en suelos derivados de ceniza volcánica, dado a la presencia de alófano el cual puede fijar formas orgánicas del S (Blair y Lefroy, 1987). Los trabajos de Blair y Lefroy también indicaron que suelos me teorizados tienen capacidades de intercambio aniónicas más altas en comparación a suelos de climas templados y en si una capacidad para adsorción de sulfato. Los experimentos conducidos por Pearson et al. (1962) demostraron que el 90\% de to-

\section{ABSTRACT5}

Two field experiments were established in 1988 at Opico Quezaltepeque, El Salvador, on volcanic-ash derived soils to evaluate maize and bean grain yield response to applied sulfur, calcium and potasium. Also under evaluation was the effect of burning crop residues (common practice in this area) compared to zero tillage. The common system employed in this region is a maize-bean relay whereby the maize is planted in May and the beans in August. Four cycles of data were obtained at both locations ( 2 Maize, 2 beans). The treatments consisted of various combinations of ammonium sulfate and urea with and without sulfur, calcium and potasium.

Grain yields were higher when ammonium sulfate (100 kg N/ha) was joint band applied with formula (16-20-0, $30 \mathrm{~kg} \mathrm{P}_{2} \mathrm{O}_{5}$ ha) under zero tillage. When this same treatment was evaluated when residues were burned, grain yields were significantly lower. When comparing ammonium sulfate snd urea jointly applied with phosphorus a possible response to sulfur was noted on these soils. Aplications of potasium did not increase grain yields of either maize or beans. When dolomitic limestone was applied at arate of 3 tons/ha, no significant differences were found in maize grain yields, however, a significant bean yield increase was found the second year compared with the no lime treatment.

das las bases solubles en agua fueron lixiviadas como sulfato en los suelos ácidos estudiados. Esto indica una baja adsorción de iones de sulfato en el complejo de intercambio aniónico.

La importancia del alófano en suelos derivados de ceniza volcánica en relación a la capacidad de fijación de P y S, fue discutido por Ishizuka y Black (1977). En este trabajo notaron que el alófano tiene la tendencia para que aluminio sea activo a pH bajo. Con el seguimiento de la meteorización, la lixiviación de bases y sílica hace que el complejo del suelo sea dominado por óxidos reactivos de aluminio $(\mathrm{Al})$ y hierro (Fe) (Blair, 1988). Otros trabajos en suelos volcánicos han demostrado que aplicaciones de $\mathrm{P}$ y $\mathrm{K}$ produjeron deficiencias de magnesio (Mg) (Ishizuka y Black, 1977).

${ }^{1}$ Trabajo presentado en la XXXVI Reunión Anual del PCCMCA, San Salvador, El Salvador, marzo 1990.

2 Técnico del Departamento de Validación, CENTA.

3 Técnico, Departamento de Suelos, CENTA.

${ }^{4}$ Agrónomo Regional, CIMMYT.

${ }^{5} \mathrm{El}$ abstract es traducción del compendio.

Publicado en Agronomía Mesoamericana, Vol. 2 (1991). 
Cuadro 1. Fertilizantes aplicados a la siembra en el cultivo de maíz, Opico-Quezaltepeque, El Salvador, $1988,1989$.

\begin{tabular}{|c|c|c|c|c|c|c|c|c|c|}
\hline Tratamiento & $\begin{array}{l}\text { Urea, } \\
\mathrm{kg} \mathrm{N} \mathrm{ha}^{-1}\end{array}$ & $\begin{array}{l}\text { Sulfato de } \\
\text { amonio, } \\
\mathrm{kg} \mathrm{N} \mathrm{ha}^{-1}\end{array}$ & $\begin{array}{l}\text { 16-20-0, } \\
\text { kg N ha }^{-1}\end{array}$ & $\begin{array}{l}\text { Sulfato de } \\
\text { amonio, } \\
\mathrm{kg} \mathrm{S} \mathrm{ha}^{-1}\end{array}$ & $\begin{array}{l}\text { Sulfato de } \\
\text { potasio,* } \\
\mathrm{kg} \mathrm{S} \mathrm{ha}^{-1}\end{array}$ & $\begin{array}{c}\mathrm{CaMg}\left(\mathrm{CO}_{3}\right)_{2} \\
\mathrm{~kg} \mathrm{Mg} \mathrm{ha}^{-1}\end{array}$ & $\begin{array}{c}16-20-0 \\
\mathrm{~kg} \mathrm{P}_{2} \mathrm{O}_{5} \mathrm{ha}^{-1}\end{array}$ & $\begin{array}{l}\text { Sulfato de } \\
\text { potasio, }{ }^{* *} \\
\text { kg K ha }^{-1}\end{array}$ & Labranza \\
\hline SA C & & 26 & 24 & 29.7 & & & 30 & & Conser. \\
\hline SA Q & & 26 & 24 & 29.7 & & & 30 & & Quema \\
\hline UR C & 26 & & 24 & & & & 30 & & Conser. \\
\hline UR C Ca & 26 & & 24 & & & 3 & 30 & & Conser. \\
\hline UR C SP & 26 & & 24 & & 29.7 & & 30 & 36 & Conser. \\
\hline UR C SP Ca & 26 & & 24 & & 29.7 & 3 & 30 & 36 & Conser. \\
\hline Agricultor & & & 46 & & & & 57 & & \\
\hline
\end{tabular}

*se aplico $\mathrm{CaSO}_{4}$ en 1989

** no se aplico K en 1989

Aunque la labranza cero es una alternativa para ayudar en el control de la erosión, trabajos hechos por Blevins et al. (1977) han demostrado que esta práctica en suelos ácidos puede resultar en una acidez mayor en el horizonte del suelo superficial.

Los objetivos principales que motivaron el establecimiento de estos experimentos fueron: medir el efecto de azufre y calcio en la producción de grano de maíz y detenninar su efecto residual en el frijol (Phaseolus vulgaris L.) de relevo, evaluar un nivel más bajo de $\mathrm{P}$ en comparación a lo que aplica el agricultor y observar algunos efectos de la

Cuadro 2. Fertilizantes aplicados treinta días después de la siembra en el cultivo de maíz, Opico Quezaltepeque, El Salvador, 19881989.

\begin{tabular}{|c|c|c|c|c|c|}
\hline Tratamiento & $\begin{array}{c}\text { Urea, } \\
\mathrm{kg} \mathrm{N} \mathrm{ha}^{-1}\end{array}$ & $\begin{array}{l}\text { Sulfato de } \\
\text { amonio, } \\
\text { kg N ha }{ }^{1}\end{array}$ & $\begin{array}{l}\text { Sulfato de } \\
\text { amonio, } \\
\mathrm{kg} \mathrm{S} \mathrm{ha}^{-1}\end{array}$ & $\begin{array}{l}\text { Sulfato de } \\
\text { potasio, } \\
\mathrm{kg} \mathrm{S} \mathrm{ha}^{-1}\end{array}$ & $\begin{array}{l}\text { Sulfato de } \\
\text { potasio, } \\
\mathrm{kg} \mathrm{K} \mathrm{ha}^{-1}\end{array}$ \\
\hline
\end{tabular}

\begin{tabular}{llllll} 
SA C & & 50 & 57.1 & & \\
SA Q & & 50 & 57.1 & & \\
UR C & 50 & & & & \\
UR C Ca & 50 & & & 57.1 & 69.4 \\
UR C SP & 50 & & & 57.1 & 69.4 \\
UR C SP Ca & 50 & & & \\
Agricultor & & 60 & 68.4 & & \\
\hline
\end{tabular}

quema en el sistema comparado con la labranza cero.

\section{MATERIALES Y MÉTODOS}

Maíz fue cultivado en cerros de Opico Quezaltepcque, El Salvador, en 2 ensayos que fueron seguidos por dos años consecutivos, en los cuales habían dos ciclos por año, un culti vo de maíz y uno de frijol. Los tratamientos consistieron en varias combinaciones de fósforo $(\mathrm{P})$, fuentes de nitrógeno $(\mathrm{N})$, cal dolomítica, sulfato de potasio y sulfato de calcio. Estos tratamientos son definidos en los Cuadros $1 \mathrm{y}$ 2. El diseño utilizado en el campo fue de bloques completos al azar.

Los suelos de esta área han tenido la influencia de ceniza volcánica y tienen pendientes que varían de 18 a 50\%. En los últimos 50 años, esta área se ha caracterizado por tener una producción continua de maíz (sembrado en mayo) y frijol (sembrado en relevo en agosto) por pequeños agricultores (área promedio de 2.0 ha). El área también es caracterizada por las quemas anuales de residuos de las cosechas de estos cultivos y pérdidas de suelo por causa de lo anterior. La quema en este ensayo se refiere a la eliminación de los residuos de la cosecha anterior con fuego. Muestras de suelo por localidad fueron tomadas antes de iniciar los estudios y los resultados se presentan en el Cuadro 3.

Las malezas en los ensayos fueron controladas con

Cuadro 3 Características químicas de los suelos de Opico Quezaltepeque, EI Salvador, Mayo $1988,1989$.

\begin{tabular}{|c|c|c|c|c|c|c|c|c|c|c|c|}
\hline Localidad & Año & $\mathrm{pH}$ & $P, u g g^{-1}$ & $\begin{array}{c}\mathrm{K}, \\
\mathrm{meq} / 100 \mathrm{~g}\end{array}$ & $\begin{array}{c}\mathrm{Ca}, \\
\mathrm{meq} / 100 \mathrm{~g}\end{array}$ & $\begin{array}{c}\mathrm{Mg}, \\
\mathrm{meq} / 100 \mathrm{~g}\end{array}$ & M.O. \%, & Mn, ug $\mathrm{g}^{-1}$ & $\mathrm{Zn}$, ug $\mathbf{g}^{-1}$ & $\mathrm{Fe}, \mathrm{ug} \mathrm{g}^{-1}$ & $\mathrm{~S}, \mathrm{ug} \mathrm{g}^{-1}$ \\
\hline Ascencio & 88 & 5.7 & 41 & 200 & 5.8 & 1.2 & 2.6 & 1.8 & 4.8 & 45.0 & \\
\hline Ascencio & 89 & 5.5 & 42 & 200 & 5.6 & 1.1 & 3.3 & 18.6 & 14.2 & 23.7 & 11.6 \\
\hline Martinez & 88 & 5.6 & 17 & 200 & 7.9 & 1.5 & 3.9 & 29.2 & 9.2 & 29.6 & \\
\hline Martinez & 89 & 5.6 & 7 & 200 & 10.9 & 2.9 & 4.2 & 23.2 & 14.5 & 13.3 & 11.3 \\
\hline
\end{tabular}

P, K - Nelson et al., (1953)

Ca, Mg - Extracción, $1 \mathrm{~N} \mathrm{KCl,} \mathrm{absorción} \mathrm{atómica}$

M.O. - Materia orgánica, por Walkley y Black, (1934).

\$ - Fox et al. (1964). Mn, Zn. Fe, absorción atómica. 
aplicaciones anuales de Atrazina (2-Cloro-4 etiloamina-6 isopropilamina-1, 3, 5 triazina) a una dosis de $1.2 \mathrm{~kg}$ i. a./ha. Las plagas del suelo (Phyllophaga spp., y Ulus spp.) se controlaron con aplicaciones anuales junto a la siembra del maíz, con Lorsban 2.5 granular a una dosis de $32 \mathrm{~kg}$ / ha Las plagas del follaje (Diabrotica spp.,y Spodoptera spp.) en el maíz y en el frijol fueron controladas con aplicaciones manuales de Tamaron 600 a una dosis de 1.0 l/ha. Un híbrido de maíz del Programa Nacional de El Salvador (CENT A), R-5 fue sembrado a una profundidad de $5 \mathrm{~cm}$ en mayo de 1988 y 1989, a una población de 55,000 plantas/ha. Las parcelas consistieron en cuatro surcos a $0.80 \mathrm{~m}$ y de $5.0 \mathrm{~m}$ de largo. Distancia entre posturas fue $0.40 \mathrm{~m}$. El frijol, variedad Rojo de Seda fue sembrado en relevo en agosto de 1988 y 1989, a una población de 200,000 plantas/ha. Dos surcos de frijol fueron sembrados por cada surco de maíz, con una distancia de $0.25 \mathrm{~m}$ al cuadro.

En esta región se reciben 1600 m de precipitación promedio anual con $90 \%$ de esta cantidad concentrada en los meses de mayo a septiembre. La temperatura promedio anual es de $25^{\circ} \mathrm{C}$ y las áreas estudiadas tenían una elevación de $450 \mathrm{~m}$ sobre el nivel del mar. La textura general de los suelos de esta área es Franco arenosa.

Los fertilizantes fueron aplicados al momento de la siembra de maíz juntos en postura incorporada $7.0 \mathrm{~cm}$ al lado del surco y $5.0 \mathrm{~cm}$ debajo de la superncie del suelo. Nitrógeno fue aplicado fraccionado en el maíz (medio ala siembra y medio a los 30 días). Los otros fertilizantes y tratamientos definidos en el Cuadro 1 fueron aplicados a la siembra menos la cal dolomítica que se aplicó 8 días antes y se incorporó en forma mecánica con el suelo. En 1988, se aplicaron 23 $\mathrm{kg} \mathrm{N} /$ hay $12.2 \mathrm{~kg} \mathrm{P} /$ ha como 16-20-0 al momento de la siembra, en postura superficial. En 1989, se aplicó $40 \mathrm{~kg} \mathrm{~N} /$ ha como urea incorporado al momento de la siembra al frijol sin ninguna aplicación de P para observar el efecto residual de los tratamientos con P utilizados en el maíz.

El testigo del agricultor representaba lo común del área relativo a fertilizantes, fuentes y dosis aplicadas. Todo lo demás (control de insectos, malezas, etc.) se trató igual a los otros tratamientos en el ensayo. Aún el tratamiento del agricultor en estos dos ensayos, el manejo fue bajo labranza cero.

Cada ciclo, el grano de maíz y frijol fue cosechado a mano de los surcos centrales y sub muestreado para humedad. De esta misma área se determinó población final a la cosecha. Se combinaron datos de frijol y de maíz por separado (Maíz 88 y Maíz 89, Frijol 88, Frijo1 89) lo cual permitió el análisis en un modelo combinado por localidad tomando en cuenta el factor año como parcelas divididas en tiempo. Análisis de covarianza por cultivo (incluyendo las dos localidades y dos años de datos) fue empleado utilizando población de plantas al momento de la cosecha como covariable ya que se determinó que la población no fue significativa como variable dependiente en relación a los tratamientos en el ensayo.

\section{RESULTADOS Y DISCUSION}

El análisis utilizado para la discusión de los resultados, separa maíz y frijol en análisis independientes (Cuadro 4). Como habían dos años de datos los cuales venían del mismo sitio, se requería un análisis de parcelas divididas en tiempo. Mediante el análisis de covarianza (covariable $=$ población) se disminuyó la variabilidad explicada por el modelo en comparación al análisis convencional. Esto se comprobó al comparar los valores del error experimental $(0.033,0.022,0.58$ y 0.56, análisis de varianza, análisis de covarlanza para frijol y maíz respectivamente). En los dos casos, maíz y frijol, no había interacciones de efectos principales (Tratamiento, Año, Localidad) significativos en el Cuadro 4. Independientemente, los efectos principales de tratamientos y año fueron significativos para el análisis combinado de maíz y el de frijol. La discusión de resultados se enfoca a las medias por tratamiento y ciclo que se presentan en el Cuadro 5 . El efecto de año se interpreta simplemente en que los rendimientos de maíz y de frijol fueron más altos en 1989 en comparación a los de 1988.

Cuadro 4 Análisis de covarianza combinando ciclos de maíz y frijol por separado, Opico Quezaltepeque, El Salvador, 1988-1989.

\begin{tabular}{|c|c|c|c|c|c|}
\hline \multirow[t]{2}{*}{ Fuente de Variación } & \multirow{2}{*}{$\begin{array}{l}\text { Grados de } \\
\text { Libertad }\end{array}$} & \multicolumn{4}{|c|}{ Cuadrados Medios } \\
\hline & & Maíz 88, Maíz & 89 & Frijol 88, Frijo & ol 89 \\
\hline Localidad & 1 & 24.65 & & 0.23 & \\
\hline (A) Loacalidad(Rep) & 4 & 1.06 & & 0.15 & \\
\hline Tratamiento & 6 & 0.89 & $*$ & 0.08 & $* *$ \\
\hline Localidad*Tratamiento & 6 & 0.15 & & 0.02 & \\
\hline (B) Rep*Tratamiento(Localidad) & 24 & 0.28 & & 0.01 & \\
\hline Año & 1 & 4.65 & $*$ & 0.39 & $* *$ \\
\hline Localidad*Año & 1 & 0.46 & & 0.01 & \\
\hline Tratamiento*Año & 6 & 0.70 & & 0.03 & \\
\hline \# Poblacion & 1 & 1.10 & & 0.38 & \\
\hline (C) Residual & 33 & 0.56 & & 0.02 & \\
\hline
\end{tabular}

En esta zona es común la quema de los residuos vegetales del maíz y frijol la que por ser en terrenos de ladera induce a la erosión del suelo. En el caso de maíz, había reducción del rendimiento debido a la quema de 0.68 y $0.28 \mathrm{Mg}$ / ha en 1988 y 1989 respectivamente (Cuadro 5, Trat 1 vs.2). Normalmente la mayor parte del azufre de fuentes orgánicas es volatilizado cuando se quema (Sánchez, 1981). Como en estos dos tratamientos (SA C y SA Q) se aplicó un total de $86.8 \mathrm{~kg} \mathrm{~S} /$ ha en dos diferentes épocas. difícilmente se atribuyen las diferencias observadas a la carencia de este elemento. Los rendimientos del frijol fueron más bajos cuando se quemaba $(0.09$ y $0.16 \mathrm{Mg}$ / ha para 1988 y 1989 respectivamente). Considerando que estos dos tratamientos también tenían $\mathrm{P}$ aplicado en cantidades suficientes, es difícil explicar estas diferencias. Una posible explicación estaría 
Cuadro 5 Rendimiento en tha por ciclo, combinando localidades, Opico Quezaltepeque, EI Salvador 1988-89.

\begin{tabular}{|c|c|c|c|c|c|c|}
\hline \multicolumn{2}{|c|}{ No. Tratamiento } & \multirow{2}{*}{$\frac{\text { Maíz } 88}{3.95}$} & \multirow{2}{*}{ Maíz 89} & \multirow{2}{*}{$\frac{\text { Frijol } 88}{1.18}$} & \multirow{2}{*}{ Frijol 89} & \multirow{2}{*}{$\frac{\text { TOTAL }}{10.69}$} \\
\hline 1 & $\mathrm{SAC}$ & & & & & \\
\hline 2 & SA Q & 3.27 & 4.03 & 1.09 & 1.09 & 9.48 \\
\hline 3 & UR C & 3.42 & 4.01 & 1.17 & 1.11 & 9.71 \\
\hline 4 & UR C Ca & 3.03 & 3.65 & 1.08 & 1.21 & 8.97 \\
\hline 5 & UR C SP & 3.69 & 3.42 & 1.08 & 1.14 & 9.33 \\
\hline 6 & UR C SP Ca & 3.79 & 3.30 & 1.26 & 1.39 & 9.74 \\
\hline 7 & Agricultor. & 3.73 & 4.27 & 1.19 & 1.07 & 10.26 \\
\hline \multirow{2}{*}{\multicolumn{2}{|c|}{$\begin{array}{l}\text { MEDIA } \\
\text { LSD }(0.10)\end{array}$}} & 3.55 & 3.85 & 1.15 & 1.18 & \\
\hline & & 0.51 & & 0.11 & & 0.89 \\
\hline
\end{tabular}

en que bajo labranza de conservación, los residuos sobre la superficie del suelopodiian haber incrementado la cantidad de agua disponible durante el ciclo en razón de una menor evaporación directa comparado con el suelo descubierto y sin residuo. Más aún, visualmente fue observado que había mayor acame en las parcelas quemadas comparadas con las de labranza cero, aunque la disminución de los rendimientos debido al acame no fue cuantificada.

Los tratamientos (SA C y UR C) fueron diseñados para evaluar la respuesta del azufre en el sistema maíz-frijol (SA C tenía un total de $86.8 \mathrm{~kg} \mathrm{~S} /$ ha en comparación a 0 en UR C, niveles de $\mathrm{N}$ y $\mathrm{P}$ constante para los dos). Aunque parte de este efecto se podría atribuir a la fuente de $\mathrm{N}$ utilizada, se observaron incrementos significativos en el rendimiento de maíz y frijol cuando estos tratamientos se evaluaron bajo labranza cero (0.53, 0.32, - 0.01 y 0.14 para 1988 y 1989 respectivamente).

Tomando en cuenta que el uso de sulfato de amonio tiende a aumentar la acidez del suelo, el sulfato de potasio como fuente de $\mathrm{S}$ fue evaluado utilizando urea como fuente de $\mathrm{N}$. Desafortunadamente, este efecto esta confundido con la aplicación de altas cantidades de $\mathrm{K}$, las que por efecto de su concentración (acción de masa), podrían desplazar Al del complejo de cambio del suelo, el cual incrementaría la fijación del $\mathrm{P}$ y disminuiría la disponibilidad de $\mathrm{P}$ causando deficiencia (Bray, 1942). Comparando tratamientos UR C con UR C SP, a una dosis de $\mathrm{P}$ constante, se observó que la respuesta de $\mathrm{S}$ que se quería medir no estaba presente. Esto se podría atribuir al efecto negativo del rendimiento por aplicaciones de $\mathrm{K}$ debido a las razones anteriormente mencionadas.

El efecto de aplicar cal dolomíticasemuestraal comparar UR C Ca y UR C SP con UR C SP Ca. En los primeros tres ciclos (Maíz 88, Frijol 88, Maíz 89) no había respuesta significativa a los $3 \mathrm{Mg}$ de cal dolomítica / ha que se aplicó en cada ciclo. Aún, en el último ciclo de frijol de 1989, la respuesta si se observó. Tomando en cuenta la acidez de estos suelos no es sorprendente la razón del porqué la cal tardo algún tiempo para mostrar un efecto de beneficio.

U $n$ aspecto importante de esta investigación fue comparar el nivel de $\mathrm{P}$ aplicado por el agricultor (57 $\mathrm{kg} \mathrm{P}_{2} \mathrm{O}_{5} / \mathrm{ha}$ ), el cual se considera excesivo. Comparando los rendimientos en el Cuadro 5 con la aplicación del agricultor se nota que había mayor eficiencia en el uso del $\mathrm{P}$ con el nivel propuesto $\left(30 \mathrm{~kg} \mathrm{P}_{2} \mathrm{O}_{5} /\right.$ ha contra agricultor, $\left.57 \mathrm{~kg} \mathrm{P}_{2} \mathrm{O}_{5} / \mathrm{ha}\right)$ dado los rendimientos similares de estos dos tratamIentos.

Al realizar el análisis económico en el cual se involucra costos de producción e ingreso neto de cada uno de los tratamientos, se detecta que el más rentable dentro del sistema es aquel que combina labranza de conservación, con las dosis en $\mathrm{kg} /$ ha de 100 de $\mathrm{N}$ y $30 \mathrm{P}_{2} \mathrm{O}_{5}$ (Sulfato de amonio y 16-20-0) con una taza marginal de retorno del $25 \%$.

\section{CONCLUSIONES}

Como respuesta a la investigación realizada se puede concluir que los mejores rendimientos de grano fueron cuando se aplicó sulfato de amonio (100 kg N/ ha) junto con fórmula (16- 20-0, $30 \mathrm{~kg} \mathrm{P}_{2} \mathrm{O}_{5}$ / ha) en postura incorporada bajo labranza de conservación. Este mismo tratamiento con quema de residuos resultó en rendimientos significativamente más bajos (inferior en 13\%). Comparando sulfato de amonio y urea, aplicados juntos con el fósforo, se notó un incremento en rendimiento de grano, quizá debido a la aplicación de Azufre en estos suelos (Superior al 10\%). Se observó una leve tendencia del rendimiento a disminuir con las aplicaciones de sulfato de potasio tanto en maíz (1989) como en frijol (1988); sin embargo, este efecto fue significativo $(\mathrm{P} \leq 0.1)$ sólo en maíz en 1989. La respuesta de haber aplicado 3 t/ha de cal dolomítica en los dos ciclos de maíz, resultó en incrementos en el rendimiento del frijol; (superior en $9 \%$ ) pero esto se observó hasta el último ciclo.

Económicamente el tratamiento más rentable, dentro del sistema es el que combina labranza de conservación, con las dosis en $\mathrm{kg} /$ ha de $100 \mathrm{~N}$ y 30 de $\mathrm{P}_{2} \mathrm{O}_{5}$ (fuentes Sulfato de Amonio y 16-20-0), con una tasa marginal de retorno del $25 \%$.

\section{BIBLIOGRAFÍA}

BARROW, N.J. 1969. Effects of adsorption of sulfate by soils on the amount of sulfate present and its availability to plants. Soil Sci. 108:193-201.

BLAIR. OJ., ; LEFROY, R.D.B. 1987. Sulphur cycling in tropical soils and the agronomic impact of increasing use of $\mathrm{S}$ free fertilizers, increased crop production and burning of crop residues. En Proceedings of the symposium on fertilizer sulphurrequirements and sources in developing countriesof Asia and the Pacific. The Sulphur Institute, Washington D.C.

BLAIR, O.J. 1988. Chemistry of S and S cyc1e in the tropics. In Sulphur in Indian Agriculture, TSI-FAI Symposium. The Sulphur Institute, Washington D.C.

BLEVINS, R.L.; THOMAS G.W.; CORNELIUS P.L. 1977. Influence of no-tillage and nitrogen fertilization on certain soil properties after 5 years of continuous como Agron. J. 69:383-386. 
BRAY, R.H. 1942. Ionic competition in base exchange reactions. J. Am. Chem. Soc. 64: 954-963.

ENSMINGER. L.E. 1954. Some factors affecting the adsorption of sulfate by Alabama soils. Soil Sci. Soc. Am. Proc. $18: 259.264$

FITTS, J. W. 1970. Sulfur deficiency in latin america. Sulphur Institute J.. 5: 14-16.

FOX, R.L.; OLSON R.A.; RHOADESH, F. 1964. Evaluating the sulfur status of soils by plants and soil tests. Soil Sci. Soc. Proc. 28: 243-246.

ISHIZUKA. YOSHIAKI; BLACK y C.A. 1977. Soils derived from volcanic ash in Japan. Centro Internacional de Mejoramiento de Maíz y Trigo (CIMMYTI. Apdo. Postal 6-641. México D.F. 06600.

KAMPRATH, EJ.; NELSON W.L.: FITTS, J.W. 1956. The effect of $\mathrm{pH}$ sulfate and phosphate concentrations on the adsorption of sulfate by soils. Soil Sci. Soc. Am. Proe. 20:463-466
MENDOZA, V.M.; SOSA, J.H. 1983. Desarrollo de la Validación Transferencia deTecnología en el Sistema Maíz-Frijol en el área Opico-Quezaltepeque. Región II. CENTA, EI Salvador. C.A. 30 p.

NELSON. W.L.: MEHLICH A.; WINTERS. E. 1953. The development, evaluation. and useof soil tests for phosphorus availabílity. Agronorny 4: 153-188.

PEARSON. R:W.; ABRUNA, F.; CHNDLER. J. Vicente. 1962. Effect oflime and nitrogen. applications on downward movement of calc.íum and magnesium in two humic tropical soils of Puerto Rico. Soil Sci. 93:77-82. .

SANCHEZ. P.A. 1981. Suelos del trópico, características y Manejo. Instituto Interamericano de Cooperación para la Agricultura (IICA). San José., Costa Rica.

WALKLEY, A.; BLACK, I.A. 1934. An examination of the Degtjareff method for determining soil organic matter and a proposed modification of the chromic acid titration method. Soil Sci. 37:29-30. 